\title{
Camera Calibration Based on 3D-Point-Grid *
}

\author{
X.-F. Zhang ${ }^{1}$, A. Luo ${ }^{2}$, W. Tao ${ }^{1}$ and H. Burkhardt ${ }^{3 \dagger}$ \\ 1 Tech. Informatik I, Hamburg-Harburg University of Technology, 21071 Hamburg \\ 2 Mikroelektronik Anwendungszentrum (MAZ) Hamburg GmbH, 21079 Hamburg \\ ${ }^{3}$ Institut für Informatik, University of Freiburg, 79085 Freiburg \\ Germany
}

\begin{abstract}
In this paper an effective technique of camera calibration based on a $3 \mathrm{D}$-point-grid is presented. The properties of structured space points, defined as $3 \mathrm{D}$-point-grid, and their relations under perspective transformation are analyzed. Upon the correspondence between these 3D-points and their image points a calibration is made not only to compute the parameters of camera model by a linear method simply from the independent points in 3D-point-grid but also to verify, modify and regulate extrinsic parameters of the orientation and position simultaneously from the structure constraint implied in these points. On the basis of the verification, modification and regulation the intrinsic parameters of camera can be computed with more objective criteria. Experimental results show the necessity and advantage of the verification and modification of the orientation and position parameters, and prove that our calibration technique can improve the accuracy of both extrinsic and intrinsic parameters greatly.
\end{abstract}

\section{Introduction}

Camera calibration is the process of determining the internal camera geometric and optical characteristics (represented by intrinsic parameters such as focus $f$, center $C_{\mathrm{x}}, C_{\mathrm{y}}$ and scale factor $s_{\mathrm{x}}$ etc. ) and/or the $3 \mathrm{D}$ position and orientation of the camera relative to a certain world coordinate system ( called extrinsic parameters like rotation matrix $R$ and translation vector $T$ ). For a real camera this process includes also a determination of distortion parameters. A lot of papers dealing with the computation of these parameters have been published, e.g. [Tsai86], [LT88], [BM91], [LC93], [WM94], [Faug93], etc.

In most cases the extrinsic and intrinsic parameters are computed by neglecting the distortion. The intrinsic parameters can be verified by some physical methods. But the orientation and position are difficult to verify unless there are exact mechanical settings such as CMM in [BM91]. The methods in above listed

\footnotetext{
* This work was supported by DAAD for the first author and by the BMBF-project MOVIS partly

† email: x.zhang or tao@tu-harburg.d400.de, luo@maz-hh.de, or burkhardt@ informatik. uni-freiburg.de
} 
papers are based on a sequence of correspondent 3D-and $2 \mathrm{D}$-points, but no relationship exists among them, or has been considered and used. The verification of the model parameters can only be made until after all the parameters are computed.

Upon these considerations, a more effective technique, which uses structurerelated space points during calibration with the process of verification, modification and regulation, is proposed in this paper. It is proceeded as follows: (1) analysis of the structure of 3D-point-grid, its relation to the transformation parameters, its construction and location of its image counterpart; (2) improved calibration of camera parameters and results; (3) conclusions.

\section{3D-Point-Grid}

\subsection{Definition}

Suppose a sequential triple set $\mathcal{L}=\{(i, j, k), i=1, \ldots, M, j=1, \ldots, N, k=$ $1, \ldots, L\}, \Delta x, \Delta y$ and $\Delta z$ are the unit lengthes along the three orthogonal directions in space $\mathcal{R}^{3}$, then the set $\mathcal{Q}=\{(i \Delta x, j \Delta y, k \Delta z),(i, j, k) \in \mathcal{L}\}$ is defined as a $3 \mathrm{D}$-point-grid, $\mathcal{L}$ is its label set. When $\left(I_{0}, J_{0}, K_{0}\right) \in \mathcal{L}$ is chosen as the origin of a coordinate system, $\left\{\left(\left(i-I_{0}\right) \Delta x,\left(j-J_{0}\right) \Delta y,\left(k-K_{0}\right) \Delta z\right),(i, j, k) \in \mathcal{L}\right\}$ is the coordinates set of $\mathcal{Q}$.

\subsection{Characteristics in structure}

Coplanar and parallel relation Let $i=I_{1}$, a fixed number, then the elements in subset $q_{I_{1}}=\left\{\left(I_{1} \Delta x, j \Delta y, k \Delta z\right), j=1, \ldots, N, k=1, \ldots, L\right\}$ are coplanar. The $M$ planes $\pi_{I_{1}}$, formed by $q_{I_{1}}\left(I_{1}=1, \ldots, M\right)$, are parallel, and $\mathcal{Q}=\cup_{I_{1}=1}^{M}\left(q_{I_{1}}\right)$.

Coplanar and perpendicular relation Let $i=I_{1}$ and $j=J_{1}$, then we get two subsets $q_{I_{1}}=\left\{\left(I_{1} \Delta x, j \Delta y, k \Delta z\right), j=1, \ldots, N, k=1, \ldots, L\right\}$ and $q_{J_{1}}=\{$ $\left.\left(i \Delta x, J_{1} \Delta y, k \Delta z\right), i=1, \ldots, M, k=1, \ldots, L\right\}$ respectively. They form two planes $\pi_{I_{1}}$ and $\pi_{J_{1}}$ with $\pi_{I_{1}} \perp \pi_{J_{1}}$. In the same way we can get $q_{K_{1}}$ and its $\pi_{K_{1}}$, and $\pi_{J_{1}} \perp \pi_{K_{1}}$ or $\pi_{K_{1}} \perp \pi_{I_{1}}$.

\subsection{The relations among parameters of orientation and position from 3D-point-grid}

Between subsets of the coplanar points that form parallel planes Generally, if any coordinate system takes a point in $3 \mathrm{D}$-point-grid, that is $\Delta x_{\mathrm{w}}, \Delta y_{\mathrm{w}}$ and $\Delta z_{\mathrm{w}}$ away from its previous origin in $x, y$ and $z$ direction respectively, as its current origin and is acquired by translation in three directions, we have

$$
R_{1}=R_{0}, \quad T_{1}=T_{0}+R_{0}\left(\Delta x_{\mathrm{w}}, \Delta y_{\mathrm{w}}, \Delta z_{\mathrm{w}}\right)^{T}
$$

in which $\Delta y_{\mathrm{w}}=\Delta z_{\mathrm{w}}=0$ corresponds to the situation of parallel plane in $\mathrm{x}$ direction, while $\Delta x_{\mathrm{w}}=\Delta z_{\mathrm{w}}=0, \Delta x_{\mathrm{w}}=\Delta y_{\mathrm{w}}=0$ to $\mathrm{y}$ and $\mathrm{z}$ direction respectively. 
Between subsets of coplanar points that form perpendicular planes Suppose that an origin is selected with a label $\left(I_{0}, J_{0}, K_{0}\right)$, then (see Fig. 1(a)) $q_{I_{0}}=\left\{\left(I_{0} \Delta x, j \Delta y, k \Delta z\right), j=1, \ldots, N, k=1, \ldots, L\right\}$ forms $\pi_{I_{0}}$ i.e. $y_{0}-O-z_{0}$ plane $q_{J_{0}}=\left\{\left(i \Delta x, J_{0} \Delta y, k \Delta z\right), i=1, \ldots, M, k=1, \ldots, L\right\}$ forms $\pi_{J_{0}}$ i.e. $x_{0}-O-z_{0}$ plane $q_{K_{0}}=\left\{\left(i \Delta x, j \Delta y, K_{0} \Delta z\right), i=1, \ldots, M, j=1, \ldots, N\right\}$ forms $\pi_{K_{0}}$ i.e. $x_{0}-O-y_{0}$ plane in $x_{0}-y_{0}-z_{0}$ coordinate system. Suppose that different coordinate systems

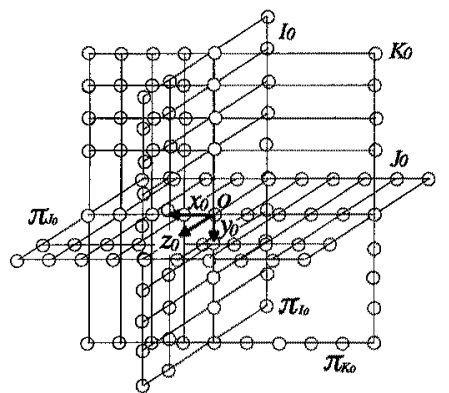

(a)
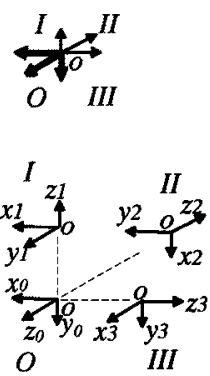

(b)

Fig. 1. (a)Points on perpendicular planes and (b) Selection of coordinate systems

are selected on these three planes by rules: (1) the origins are the same as that of $x_{0}-y_{0}-z_{0} ;(2)$ all are right-handed systems; (3) all $x-0-y$ planes are on these three planes; (4) all $z$ axes are in counter direction of the axes of $x_{0}-y_{0}-z_{0}$, as shown in Fig. 1(b). The rotation matrices of these coordinate systems, which transform points in coordinate systems O, I, II, III into camera coordinate system, are supposed to be $R_{0}, R_{1}, R_{2}, R_{3}$, then

(i) if $R_{i j}$ stands for a matrix that transforms a point $\left(x_{i}, y_{i}, z_{i}\right)$ from $i$ th coordinate system into $j$ th coordinate system, then the matrices for the coordinate transformations among O, I, II and III are listed in Table 1.

(ii) if a point in coordinate system $I$ is transformed into coordinate system $O$, and then to camera coordinate system, then the rotation matrices have relations $R_{1}=R_{0} R_{10}$. Similarly we have $R_{1}=R_{2} R_{12}, R_{1}=R_{3} R_{13}$. Same relations hold for other coordinate systems and can be concluded in Table 2(a).

\subsection{Construction of 3D-Point-Grid and extraction of image points}

In experiment a 3D-point-grid $\mathcal{Q}=\cup_{I_{1}=1}^{M}\left(q_{I_{1}}\right)$ is constructed by the horizontal movement of a square pattern, or specially made cube with surfaces of square patterns, as shown in Fig.2. By steps: (1) subpixel edge detection; (2) edge tracing; (3) corner detection; (4) computation of corner-coordinates (see [Zhang96] ), the image points can be extracted. 
Table 1. Transformations of the coordinate systems on perpendicular planes

\begin{tabular}{|c|c|c|c|c|c|c|c|c|}
\hline$i \nearrow j$ & & $\mathrm{O}$ & & $I$ & & II & & III \\
\hline $\mathrm{O}$ & $R_{00}$ & {$\left[\begin{array}{lll}1 & 0 & 0 \\
0 & 1 & 0 \\
0 & 0 & 1\end{array}\right]$} & $R_{01}:$ & {$\left[\begin{array}{ccc}1 & 0 & 0 \\
0 & 0 & 1 \\
0 & -1 & 0\end{array}\right]$} & $R_{02}:$ & {$\left[\begin{array}{ccc}0 & 1 & 0 \\
1 & 0 & 0 \\
0 & 0 & -1\end{array}\right]$} & $R_{03}:$ & {$\left[\begin{array}{ccc}0 & 0 & 1 \\
0 & 1 & 0 \\
-1 & 0 & 0\end{array}\right]$} \\
\hline I & $R_{10}:$ & {$\left[\begin{array}{ccc}1 & 0 & 0 \\
0 & 0 & -1 \\
0 & 1 & 0\end{array}\right]$} & $R_{11}$ : & {$\left[\begin{array}{lll}1 & 0 & 0 \\
0 & 1 & 0 \\
0 & 0 & 1\end{array}\right]$} & $R_{12}:[$ & {$\left[\begin{array}{ccc}0 & 0 & -1 \\
1 & 0 & 0 \\
0 & -1 & 0\end{array}\right]$} & $R_{13}:$ & {$\left[\begin{array}{ccc}0 & 1 & 0 \\
0 & 0 & -1 \\
-1 & 0 & 0\end{array}\right]$} \\
\hline II & $R_{20}$ & {$\left[\begin{array}{ccc}0 & 1 & 0 \\
1 & 0 & 0 \\
0 & 0 & -1\end{array}\right]$} & $R_{21}$ & {$\left[\begin{array}{ccc}0 & 1 & 0 \\
0 & 0 & -1 \\
-1 & 0 & 0\end{array}\right]$} & $R_{22}$ & {$\left[\begin{array}{lll}1 & 0 & 0 \\
0 & 1 & 0 \\
0 & 0 & 1\end{array}\right]$} & $R_{23}:$ & {$\left[\begin{array}{ccc}0 & 0 & -1 \\
1 & 0 & 0 \\
0 & -1 & 0\end{array}\right]$} \\
\hline III & $R_{30}:$ & {$\left[\begin{array}{ccc}0 & 0 & -1 \\
0 & 1 & 0 \\
1 & 0 & 0\end{array}\right]$} & $R_{31}$ & {$\left[\begin{array}{ccc}0 & 0 & -1 \\
1 & 0 & 0 \\
0 & -1 & 0\end{array}\right]$} & $R_{32}:$ & {$\left[\begin{array}{ccc}0 & 1 & 0 \\
0 & 0 & -1 \\
-1 & 0 & 0\end{array}\right]$} & $R_{33}:$ & {$\left[\begin{array}{lll}1 & 0 & 0 \\
0 & 1 & 0 \\
0 & 0 & 1\end{array}\right]$} \\
\hline
\end{tabular}

Table 2. (a) Relations of coordinates' transformations on three perpendicular planes and (b) illustration of modification of $R_{0}, R_{1}, R_{2}, R_{3}$

\begin{tabular}{|c||c|c|c|c|}
\hline$R_{i j}$ & $R_{0}$ & $R_{1}$ & $R_{2}$ & $R_{3}$ \\
\hline \hline$R_{0}$ & $R_{0} I$ & $R_{0} R_{10}$ & $R_{0} R_{20}$ & $R_{0} R_{30}$ \\
\hline$R_{1}$ & $R_{1} R_{01}$ & $R_{1} I$ & $R_{1} R_{21}$ & $R_{1} R_{31}$ \\
\hline$R_{2}$ & $R_{2} R_{02}$ & $R_{2} R_{12}$ & $R_{2} I$ & $R_{2} R_{32}$ \\
\hline$R_{3}$ & $R_{3} R_{03}$ & $R_{3} R_{13}$ & $R_{3} R_{23}$ & $R_{3} I$ \\
\hline
\end{tabular}

(a)

$$
\begin{aligned}
& R_{0} \Rightarrow R_{0} I=R_{0}^{0} \searrow \nearrow \overline{R_{0} I} \Rightarrow \bar{R}_{0} \\
& R_{1} \Rightarrow R_{1} R_{10}=R_{0}^{1} \searrow \overline{R_{0} R_{01}} \Rightarrow \bar{R}_{1} \\
& R_{2} \Rightarrow R_{2} R_{20}=R_{0}^{2} \nearrow \bar{R}_{0} \searrow \bar{R}_{0} R_{02} \Rightarrow \bar{R}_{2} \\
& R_{3} \Rightarrow R_{3} R_{30}=R_{0}^{3} \nearrow \searrow \overline{R_{0} R_{03} \Rightarrow \bar{R}_{3}}
\end{aligned}
$$

\section{Improved Camera Calibration: Verification, Modification and Regulation, and their Results}

\subsection{R's Verification and Modification}

Case of parallel planes According to (1) there should be $R_{0}=R_{1}=\ldots=$ $R_{\mathrm{M}-1}$ for $R_{j}(j=0, \ldots, \mathrm{M}-1)$ that are computed initially by Tsai's coplanar methods. In the light of this relation the validation can be verified by checking $R_{j}$ s' elements or the angles from them. A common rotation matrix can be modified by averaging the orientation angles from them.

Case of perpendicular planes The verification can be made by checking the relations listed in Table 1 . And furthermore based upon these relations, $R_{i j} \mathrm{~s}$ can be modified by the process listed in Table 2(b). The verifying results in cube-suface experiment are given in Table 3 . 

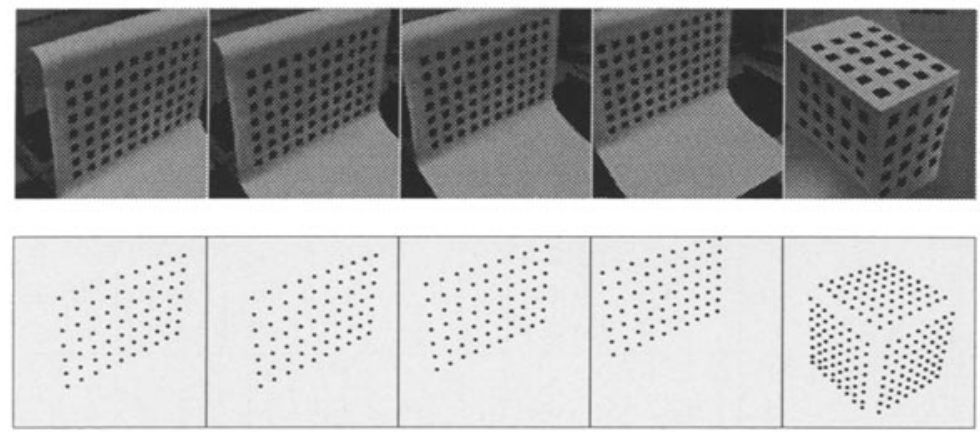

Fig. 2. Original images and the extracted corners for the construction of a $3 \mathrm{D}$-point-grid with parallel planes or surfaces of a cube.

\subsection{T's Verification and Regulation}

Case of parallel planes Considering the orthogonality of $R_{j}$ and the real geometrical relation among these coordinate systems, we have $\left|T_{(j+1)}-T_{j}\right|=$ $D_{(j+1) j}, \quad j=0, \ldots, \mathbf{M}-2$, where $D_{(j+1) j}$ are the distances between the origins of the coordinate system on these planes. These relations can serve as a verification of $T_{j}$. They can be changed into one objective function

$$
F=\left[\sum_{j=0}^{M-2}\left(\left|T_{(j+1)}-T_{j}\right|-D_{(j+1) j}\right)^{2}\right]^{1 / 2}=\min
$$

and its minimization acts as a goal to the regulation of $T_{j}$.

In our experiment the origins of the world coordinate systems are selected at the top-right corner of the top-right square (see Fig.3(a)), $D_{(j+1) j}=Z_{(j+1) j}$. The results show that $\left|T_{2}-T_{1}\right|,\left|T_{3}-T_{2}\right|$ in Table 4 are much nearer to the real distances. Figure 4 gives the shape and contour of the objective function at final iteration, in which the minimium corresponds to optimal values of $T_{i}, f$ and $C_{y}$.

Case of perpendicular planes In this case, when the coordinate systems are chosen on each plane, as shown in Fig. 3(b), if $D_{12}, D_{23}$ and $D_{31}$ stand for the distances among the origins of the selected coordinate systems, the objective function can be constructed as

$$
F=\left[\left(\left|T_{\mathrm{II}}-T_{\mathrm{I}}\right|-D_{12}\right)^{2}+\left(\left|T_{\mathrm{III}}-T_{\mathrm{II}}\right|-D_{23}\right)^{2}+\left(\left|T_{\mathrm{I}}-T_{\mathrm{III}}\right|-D_{31}\right)^{2}\right]^{1 / 2}=\min
$$

With this function the regulation is similar to that of the parallel plane. In experiment we choose $D_{12}=D_{23}=D_{31}=0$, the results are shown in Table 5 .

Finally improvements are made by optimalization of the total residiual error of all correspondent 2D- and 3D-points for the image center $\left(C_{\mathrm{x}}, C_{\mathrm{y}}\right)$. Distortion 
parameters $k_{1 \mathrm{x}}, k_{2 \mathrm{x}}, k_{1 \mathrm{y}}$ and $k_{2 \mathrm{y}}$ are computed based on Tsai's Radial Alignment Constraint model. A set of calibrated parameters are given in Table 6 .

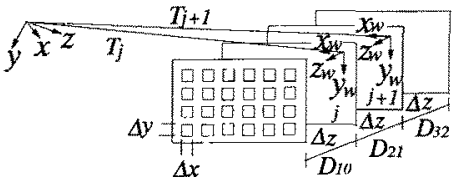

(a) Case of parallel planes

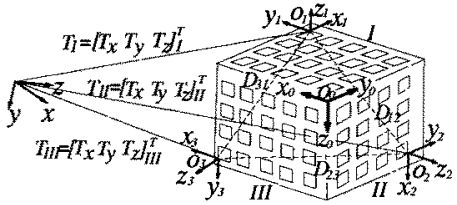

(b) Case of perpendicular planes

Fig. 3. Verification and modification of $R, T$.

\section{Conclusions}

A method of camera calibration that computes the parameters from 3D-pointgrid, a group of structure related 3D points, is proposed in this paper. According to objective criteria deduced from the 3D-point-grid the camera extrinsic parameters are verified, modified and regulated. Based on the accurate estimation, the intrinsic parameters of the camera continue to be optimized. It is proved experimentally that our method can improve the accuracy of both extrinsic and intrinsic parameters greatly. The method can be applied not only in a well-defined lab situation but also in a nature scene with a precisely made structure-pattern.

\section{References}

[Tsai86] R. Y. Tsai, An Efficient and Accurate Camera Calibration Technique for 3D Machine Vision, Proc. of IEEE Int. Conf. on Computer Vision and Pattern Recognition, Miami Beach,FL, 1986, pp 364-374

[LT88] R. K. Lenz and R. Y. Tsai, Techniques for Calibration of the Scale Factor and Image Center for high Accuracy 3D Machine Metrology, IEEE Trans. on Pattern Analysis and Machine Intelligence, Vol. 10, May 1988, pp 713-720

[BM91] E.Bruzzone and F.Magli, Calibration of a CCD Camera on a Hybrid Coordinate Measuring Machine for Industrial Meterology, SPIE, Vol. 1526, Industrial Vision Metrology (1991), pp96-112

[LC93] Jim Z. C. Lai, M. Chao, et al., The Effects of a Camera's Intrinsic Parameters on the Determination of a Coordinate Frame, Jounal of the Chinese Institute of Engineerings, Band 16 (1993), Vol. 5, pp621-630.

[WM94] G.-Q. Wei and S. -D. Ma, Implicit and Explicit Camera Calibration, Theory and Experiments, IEEE, PAMI, Vol. 16, No. 5, May 1994, pp 469-488

[Faug93] O.Faugeras, Three-Dimensional Computer Vision - A Geometric Viewpoint, MIT Press, 1993

[Zhang96] X.-F. Zhang, Untersuchung, Implementierung und Verbesserung der Kamerakalibrierung, Technical Report, TI-1, TUHH, Nov. 1996 
Table 3. Verification of $R_{i}$ 's Relations of the Coordinate System on the Cube

\begin{tabular}{|c|c|c|c|c|c|c|}
\hline$i \nearrow j$ & & O & \multicolumn{4}{|c|}{ I } \\
\hline 0 & & $R_{00}:\left[\begin{array}{lll}1 & 0 & 0 \\
0 & 1 & 0 \\
0 & 0 & 1\end{array}\right]$ & $R_{01}:$ & {$\left[\begin{array}{l}1.00 \\
0.00 \\
0.03\end{array}\right.$} & $\begin{array}{cc}0.03 & 0 \\
0.06 & 1 \\
-1.000 & 0\end{array}$ & $\left.\begin{array}{l}0.00 \\
1.00 \\
0.06\end{array}\right]$ \\
\hline I & $R_{10}:$ & {$\left[\begin{array}{ccc}1.00 & 0.00 & 0.03 \\
0.03 & 0.06 & -1.00 \\
0.00 & 1.00 & 0.06\end{array}\right]$} & & $R_{11}:$ & {$\left[\begin{array}{lll}1 & 0 & 0 \\
0 & 1 & 0 \\
0 & 0 & 1\end{array}\right]$} & \\
\hline II & $R_{20}:$ & {$\left[\begin{array}{ccc}0.02 & 1.00 & -0.06 \\
1.00 & -0.02 & 0.05 \\
0.05 & -0.06 & -1.00\end{array}\right]$} & $R_{21}$ : & $\begin{array}{c}0.05 \\
0.11 \\
-1.00\end{array}$ & $\begin{array}{c}1.00 \\
-0.06 \\
0.05\end{array}$ & $\left.\begin{array}{l}-0.05 \\
-1.00 \\
-0.11\end{array}\right]$ \\
\hline III & $R_{30}:$ & {$\left[\begin{array}{ccc}-0.08 & 0.00 & -1.00 \\
-0.05 & 1.00 & 0.01 \\
1.00 & 0.05 & -0.08\end{array}\right]$} & $R_{31}$ : & $\begin{array}{c}-0.09 \\
0.99 \\
0.10\end{array}$ & $\begin{array}{c}0.03 \\
0.11 \\
-0.99\end{array}$ & $\left.\begin{array}{l}-1.00 \\
-0.08 \\
-0.04\end{array}\right]$ \\
\hline$i \nearrow j$ & & II & & & III & \\
\hline 0 & $R_{02}:$ & $\begin{array}{ccc}0.02 & 1.00 & 0.05 \\
1.00 & -0.02 & -0.06 \\
-0.06 & 0.05 & -1.00\end{array}$ & $R_{03}:$ & {$\left[\begin{array}{c}-0.08 \\
0.00 \\
-1.00\end{array}\right.$} & $\begin{array}{c}-0.05 \\
1.00 \\
0.01\end{array}$ & $\begin{array}{c}1.00 \\
0.05 \\
-0.08\end{array}$ \\
\hline I & $R_{12}:$ & {$\left[\begin{array}{ccc}0.05 & 0.11 & -0.99 \\
1.00 & -0.06 & 0.05 \\
-0.05 & -0.99 & -0.11\end{array}\right.$} & $R_{13}:$ & {$\left[\begin{array}{c}-0.09 \\
0.03 \\
-1.00\end{array}\right.$} & $\begin{array}{c}0.99 \\
0.11 \\
-0.08\end{array}$ & $\begin{array}{c}0.10 \\
-0.99 \\
-0.04\end{array}$ \\
\hline II & & $R_{22}:\left[\begin{array}{lll}1 & 0 & 0 \\
0 & 1 & 0 \\
0 & 0 & 1\end{array}\right]$ & $R_{23}:$ & {$\left[\begin{array}{c}0.00 \\
1.00 \\
-0.02\end{array}\right.$} & $\begin{array}{l}-0.14 \\
-0.02 \\
-0.99\end{array}$ & $\begin{array}{l}-0.99 \\
0.00 \\
0.14\end{array}$ \\
\hline III & $R_{32}$ & $\begin{array}{ccc}0.00 & 1.00 & -0.02 \\
-0.14 & -0.02 & -0.99 \\
-0.99 & 0.00 & 0.14\end{array}$ & & $R_{33}$ : & {$\left[\begin{array}{lll}1 & 0 & 0 \\
0 & 1 & 0 \\
0 & 0 & 1\end{array}\right]$} & \\
\hline
\end{tabular}

Table 4. Verification of the Distances among Origins

\begin{tabular}{|c|c|c|c|c|c|}
\hline & \multirow{2}{*}{$\begin{array}{c}\text { Real } \\
\text { Distance }\end{array}$} & \multicolumn{2}{|c|}{ before regulation } & \multicolumn{2}{|c|}{ after regulation } \\
\hline & & Computed Dis. & Differences & Computed Dis. & Differences \\
\hline$\left|T_{1}-T_{0}\right|$ & 32.35 & 30.78 & -1.57 & 29.97 & -2.38 \\
\hline$\left|T_{2}-T_{1}\right|$ & 49.95 & 55.23 & 5.28 & 50.59 & 0.64 \\
\hline$\left|T_{3}-T_{2}\right|$ & 49.25 & 89.85 & 40.60 & 49.63 & 0.38 \\
\hline
\end{tabular}

Note: $\left(\sum_{i=0}^{2}\left(\left|T_{(j+1)}-T_{j}\right|-Z_{(j+1) j}\right)^{2}\right)^{1 / 2}=40.97$ (before reg.), 2.49(after reg.) 


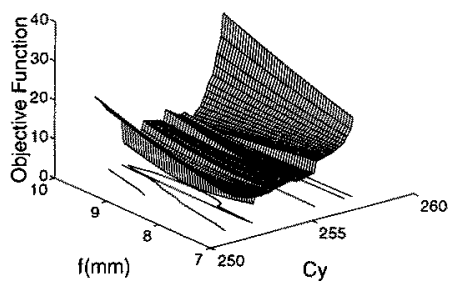

(a) Shape of the Objective Function

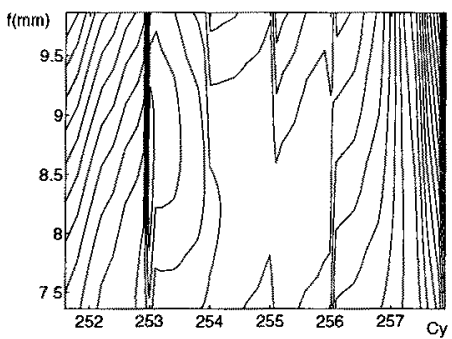

(b) Contour of the Objective Function

Fig. 4. Objective Function at the Final Iteration

Table 5. Verification of $T_{i}$ 'of the Coordinate Systems on the Cube

\begin{tabular}{|c||c|c|c|c|c|c|}
\hline unit $(\mathrm{mm})$ & $T_{I}$ & $T_{I I}$ & $T_{I I I}$ & $T_{I}-T_{I I} \mid$ & $T_{I I}-T_{I I I} \mid$ & $\left|T_{I I I}-T_{I}\right|$ \\
\hline $\bar{T}_{\mathrm{x}}$ & -0.93 & -0.11 & -0.60 & 0.82 & 0.49 & 0.32 \\
$T_{\mathrm{y}}$ & 6.57 & 6.56 & 8.61 & 0.02 & 2.05 & 2.04 \\
$T_{\mathrm{z}}$ & 272.18 & 321.87 & 249.44 & 49.69 & 72.43 & 22.74 \\
distance & & & & 49.69 & 72.46 & 22.83 \\
\hline
\end{tabular}

Before Regulation, $\left[\left(\left|T_{I I}-T_{I}\right|\right)^{2}+\left(\left|T_{I I I}-T_{I I}\right|\right)^{2}+\left(\left|T_{I}-T_{I I I}\right|\right)^{2}\right]^{1 / 2}=90.78$

\begin{tabular}{|c||c|c|c|c|c|c|}
\hline unit $(\mathrm{mm})$ & $T_{I}$ & $T_{I I}$ & $T_{I I I}$ & $\left|T_{I}-T_{I I}\right|\left|T_{I I}-T_{I I I}\right|$ & $\left|T_{I I I}-T_{I}\right|$ \\
\hline$T_{\mathrm{x}}$ & -0.93 & -0.11 & -0.60 & 0.82 & 0.49 & 0.32 \\
$T_{\mathrm{y}}$ & 6.57 & 6.56 & 8.61 & 0.02 & 2.05 & 2.04 \\
$T_{\mathrm{z}}$ & 226.26 & 226.7 & 223.12 & 0.53 & 3.67 & 3.14 \\
distance & & & & 0.97 & 4.24 & 3.76 \\
\hline
\end{tabular}

After Regulation, $\left[\left(\left|T_{I I}-T_{I}\right|\right)^{2}+\left(\left|T_{I I}-T_{I I}\right|\right)^{2}+\left(\left|T_{I}-T_{I I I}\right|\right)^{2}\right]^{1 / 2}=5.75$

Table 6. Calibrated Parameters from the Square Patterns on four Parallel Planes

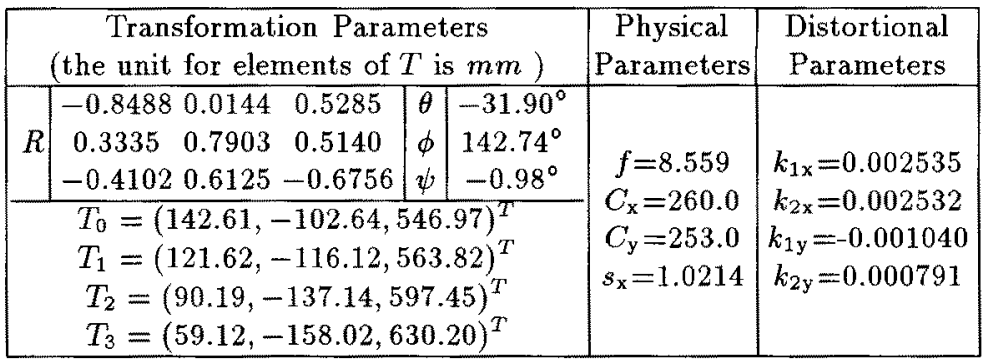

*NOTE: the selection of Vector $T_{i}$ depends on the selected world coordinate system 